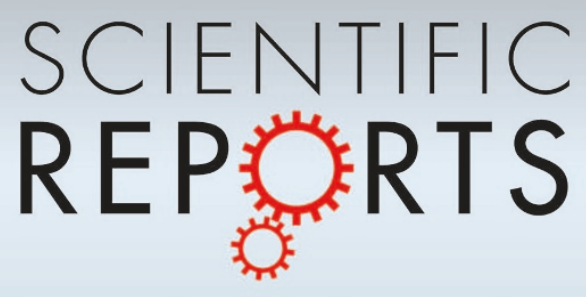

OPEN

SUBJECT AREAS:

NONLINEAR

PHENOMENA

MECHANICAL ENGINEERING

APPLIED PHYSICS

AEROSPACE ENGINEERING

Received

26 February 2013

Accepted

19 June 2013

Published

9 July 2013

Correspondence and requests for materials should be addressed to

F.P.-V.

(fpachecovazquez@ ulg.ac.be)

\section{Rebound of a confined granular material: combination of a bouncing ball and a granular damper}

\author{
F. Pacheco-Vázquez \& S. Dorbolo
}

GRASP, Physics Department B5, Université de Liège, B4000-Liège, Belgium.

A ball dropped over a solid surface bounces several times before a complete stop. The bouncing can be reduced by introducing a liquid into the ball; however, the first rebound remains largely unaffected by the fluid. Granular materials can also work as dampers. We investigated the rebound of a container partially filled with a given mass of grains $\boldsymbol{m}_{i}$. During the collision, the kinetic energy of the container is partially transferred to the grains, the rebound is damped, and the fast energy dissipation through inter-particle collisions and friction decreases the bouncing time dramatically. For grain-filled cylinders, a completely inelastic collision (zero rebound) is obtained when $m_{i} \geq 1.5 \varepsilon_{o} m_{c}$, where $\varepsilon_{o}$ and $m_{c}$ are the coefficient of restitution and mass of the empty container. For grain-filled spheres, the first rebound is almost undamped, but the second collision is completely inelastic if $m_{i} \gg m_{c}$. These findings are potentially useful to design new granular damping systems.

r he bounce of a ball dropped over a solid surface is a prolific topic in physics and engineering ${ }^{1-7}$. This simple system constitutes the basis to describe the rebound of sophisticated objects and more complex systems; for example, bouncing viscoelastic spheres ${ }^{8}$, non-homogeneous balls ${ }^{9}$, solid spheres into fluids ${ }^{10}$, balls ${ }^{11}$, dimers $^{12}$ and trimers ${ }^{13}$ on vibrated plates, and vibrated granular materials ${ }^{14-17}$.

Certain applications require materials that minimize the loss of energy during the rebound; e.g., in the design of small rubber balls in sport science ${ }^{18}$. On the other hand, in some cases it is necessary to damp the impact in order to protect the bouncing object, as in the landing of a massive spacecraft in Mars exploration missions ${ }^{19}$ (where a rover, protected by its lander structure and airbags, hits the ground and bounces several times before reaching the repose). A recent research ${ }^{20}$ showed that the rebound of a hollow sphere can be damped when it is partially filled with a liquid. The presence of the fluid does not affect the first rebound, but the second rebound is mitigated by the sloshing and collapsing of the liquid. The maximum damping is obtained when the liquid occupies about $30 \%$ of the available space and less notorious for larger liquid volumes.

Granular materials can also damp impacts and oscillations due to their ability to dissipate mechanical energy through inelastic collisions and friction between particles ${ }^{21,22}$. Recently, a considerable amount of research focused on the efficiency of granular dampers (GD) has been developed ${ }^{22-27}$. A granular damper consists of a number of grains enclosed in a receptacle that is attached or embedded in a vibrating structure ${ }^{22,26}$. In comparison with classical viscous dampers, GD exhibit several advantages for applications under extreme conditions: they are simple, easy to maintain, robust, very durable, no sensitive to temperature and effective over a wide range of frequencies ${ }^{23-25}$. It is only natural that these new dampers have a broad spectrum of applications (reducing vibrations in aerospace industry ${ }^{28,29}$, sports ${ }^{30}$, medical tools ${ }^{31}$, oscillatory saws ${ }^{32}$, dead-blow hammers ${ }^{33}$, etc). Through particle dynamics simulations ${ }^{34}$, it has been found that the damping mechanism is mainly dominated by friction for small particles, but the collisions effect becomes important as the particle size increases. However, when the number of particles is large, the total energy dissipated (collisional and frictional) is independent of the friction and restitution characteristics of the particles ${ }^{27}$. Moreover, in box-type GD, the damping efficiency is related to the available space for the grains in the container of confinement. Too confined, the grains behaves like a solid, the damping is weak. Too loose, the grains do not interact enough with the borders to damp the oscillations ${ }^{24,26}$.

In this work, we consider the damping effect of a granular material contained in a cell that freely bounces on a flat surface (i.e. the combination of a bouncing ball-and a granular damper). The main objective was to determine how the effective coefficient of restitution $(\varepsilon)$ of the charged container, as well as the bouncing dynamics, depend on the internal mass of grains. For this purpose, cylindrical containers partially filled with grains were impacted 
vertically on a solid base. Different parameters were varied: the internal mass $m_{i}$, the granular material, the length $L$, the mass $m_{c}$ and the coefficient of restitution $\varepsilon_{0}$ of the empty container, and its impact velocity. We found that $\varepsilon$ decreases linearly with $m_{i}$ to practically zero when $m_{i}=m_{\text {crit }} \sim 1.5 \varepsilon_{0} m_{c}$. Over this critical mass, the container impacts without bouncing. However, when the container is totally full of grains, it becomes "solid" and $\varepsilon$ increases notably. Our results are explained using a momentum exchange model. Moreover, we found that the container geometry plays an important role in the bouncing dynamics. In contrast with the cylindrical geometry, a grain-filled sphere never suffers a zero rebound. However, for $m_{i}$ $\gg \mathrm{m}_{\mathrm{c}}$, the second rebound is always completely inelastic, independently of the volume of grains. This latter result also contrasts with the bouncing of a fluid-filled sphere ${ }^{20}$, which damping is less effective as the internal liquid volume increases and bounces several times before the complete stop.

The experimental set-up is shown in Fig. 1a and described in the methods section. The characteristic of the containers and grain properties are summarized in Table 1 . A video illustrating the main results reported in this manuscript can be found in the supplementary material.

\section{Results}

Internal mass dependence. Figures $1(b-f)$ show snapshots of the bouncing dynamics of cylindrical containers filled with different masses of steel beads (spherical particles of $2 \mathrm{~mm}$ diameter). The parameters $\varepsilon_{0}=0.9, L=5 \mathrm{~cm}, m_{c}=10.2 \mathrm{~g}$ and $H=10 \mathrm{~cm}$ were kept constant. For the empty case $\left(m_{i}=0\right)$, a classical bouncing a)

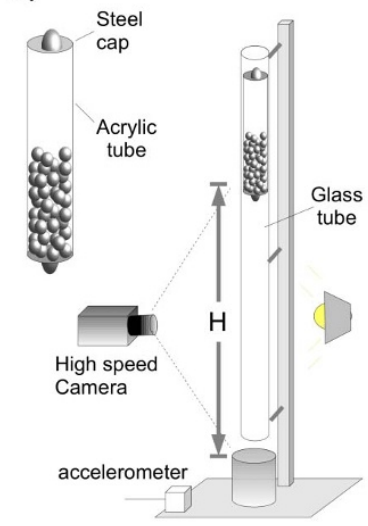

g)

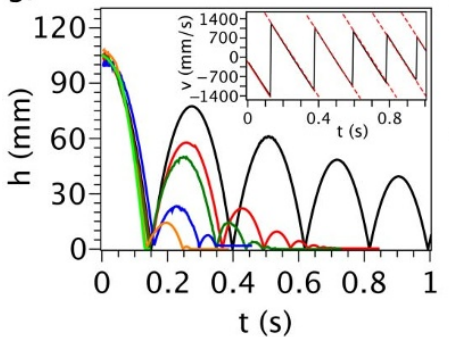

d)

e)
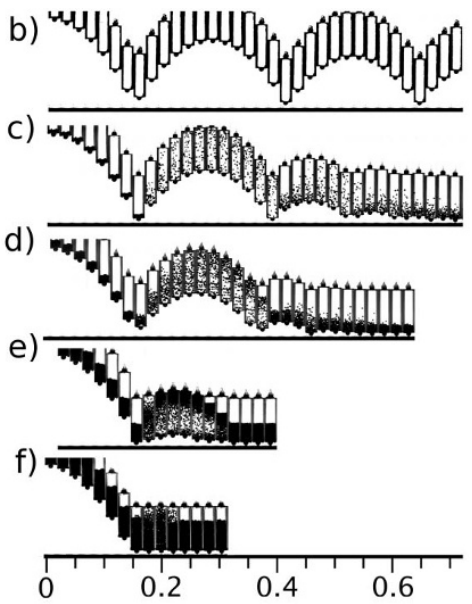

h)

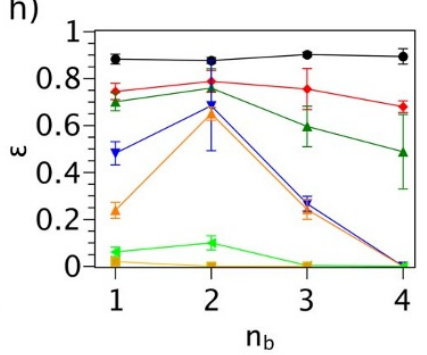

Figure $1 \mid$ (a) Sketch of the experimental set-up. (b-f) Snapshots of the bouncing cylinder $\left(m_{c}=10.5 \mathrm{~g}\right)$ with different values of inner mass $m_{i}$ : (b) empty $\left(m_{i} / m_{c}=0\right)$, (c) $1.5 \mathrm{~g}\left(m_{i} / m_{c}=0.14\right)$, (d) $3.5 \mathrm{~g}\left(m_{i} / m_{c}=0.33\right)$, (e) $10.5 \mathrm{~g}\left(m_{i} / m_{c}=1\right)$ and (f) $15 \mathrm{~g}\left(m_{i} / m_{c}=1.43\right)$. (g) Vertical position of the containers as a function of time for different values of $m_{i}$. Inset: $v v s t$ for the empty container. Red lines have a slope of $g=9810 \mathrm{~mm} / \mathrm{s}^{2}$.

(h) Restitution coefficient for the first four consecutive bounces of containers with $m_{i}=0 \mathrm{~g}(\bullet), 1.5 \mathrm{~g}(\diamond), 3.5 \mathrm{~g}(\boldsymbol{\Delta}), 7 \mathrm{~g}(\boldsymbol{\nabla}), 10.5 \mathrm{~g}(\triangleright)$, $15 \mathrm{~g}(<)$ and $18 \mathrm{~g}(\square)$. dynamics is observed (b). However, this bouncing is reduced dramatically as $m_{i}$ increases (c-e). Finally, for $m_{i}=15 \mathrm{~g}$, the container does not bounce (f). In order to characterize this bouncing dynamics, the vertical position $h$ of the geometric centre of the tube was measured and plotted as a function of time in Fig. 1g. We used the first derivative $v=d h / d t$ to obtain the vertical velocity of the container vs time. For clarity, only the empty case is plotted in the inset of Fig. 1g. This inset also shows that the air drag over the container can be neglected (red lines slope $=9810 \mathrm{~mm} / \mathrm{s}^{2}$ ). The coefficient of restitution, $\varepsilon=v_{a} / v_{b}$ (where $v_{b}$ and $v_{a}$ are the velocities before and after the impact) was plotted in Fig. 1h for the first four impacts, numbered by $n_{b}$. For the empty container (O), $\varepsilon \approx 0.9$ is independent of $n_{b}$, and therefore of the impact velocity. On the other hand, $\varepsilon$ varies considerably for grain-filled containers. For $n_{b}=1, \varepsilon$ decreases as the internal mass increases. After this initial impact, the particles go upwards faster than the container and form a cluster at the top of the cavity. For the second impact $\left(n_{b}=2\right)$, the container is "free of particles" because they are in free fall inside the cavity, thus $\varepsilon$ is maximum. Thereafter, the falling particles start to collapse on the container bottom. This collapse damps the following rebounds and reduces dramatically the coefficient of restitution. Above $m_{i}=15 \mathrm{~g}, \varepsilon \approx 0(\square)$ indicates a completely inelastic collision.

Length dependence. During the bouncing, the momentum exchange between the container and the beads takes place only through particle collisions with both ends of the container. Therefore, the length of the tube seems to be a key parameter. Figure 2 shows snapshots of the bouncing dynamics for different values of $L$ (here the parameters $\varepsilon_{0}$ $\approx 0.8, m_{i}=10.5 \mathrm{~g}, m_{c}=10.8 \mathrm{~g}$ and $H=20 \mathrm{~cm}$ were kept constant). The case $L=\infty$ corresponds to a tube without any upper end. For $L=$ $7,5,4$ and $3 \mathrm{~cm}$, the grains have enough room to move freely during the bouncing, contrary to $L=L_{\text {min }}=2.2 \mathrm{~cm}$ which corresponds to the minimum cavity size occupied for this mass of grains. The plot $h$ vs $t$ (Fig.2a) reveals that the maximum height reached by the container after the first bounce increases when $L$ is decreased. This is not due to a different value of the coefficient of restitution, which obviously is the same for the first impact. As can be observed from the snapshots, even if in all cases the initial kinetic energy of the particles is the same after the collision, in shorter tubes more particles can reach the ceiling of the cavity and transfer their energy back to the container. The transfer increases the velocity of the container and the latter rises higher. This sudden momentum exchange is reflected in the plot $v v s t$ in Fig. $2 \mathrm{~b}$ at $t \sim 0.06 \mathrm{~s}$ for closed tubes $(3-7 \mathrm{~cm})$. On the other hand, for $L_{\text {min }}=2.2 \mathrm{~cm}$, the system container-particles is almost a solid object; the particles dissipate less energy because they cannot move, and therefore the container bounces higher. Note that momentum exchange is not observed for this case in Fig. 2b (black solid line). Figure 2c shows $\varepsilon$ vs $n_{b}$ for the different containers lengths: as expected, for partially-filled containers $\varepsilon\left(n_{b}=1\right)$ has the same value, $\varepsilon$ is maximum for the second impact; and it decreases in the following rebounds due to the material collapse with the container bottom. This collapse is reflected by a sudden change in curvature of the $v$ vs $t$ plot after the second impact. In contrast, for $L=\infty$, the particles are most of the time in free flight after the first impact, they arrive to the base "one by one" and the damping is less notorious (o).

Total bouncing time $(\tau)$. Surprisingly, the time elapsed from the first impact until the container stops is largely independent of the tube length. This time, and therefore the damping efficiency, is only determined by $m_{i}$ (see Fig. $2 \mathrm{~d}$ and inset therein). By analysing the videos, we observed that this independence is expected if $L_{\text {min }}<L<$ $\left\langle v_{p}\right\rangle^{2} / 2 g$, where $\left\langle v_{p}\right\rangle$ is the average velocity of the particles after the first impact. This condition ensures that the grains can interact with the bottom and ceiling of the cavity during the first bounce and dissipate most of the initial energy of the system during these two 
Table 1 | Properties of the granular materials and containers used in the experiments. *Data measured using a free-fall ball from $\mathrm{H}=20 \mathrm{~cm}$ over solid surfaces of steel $\left(S_{b}\right)$, ceramic $\left(C_{b}\right)$ and glass $\left(G_{b}\right)$

\section{Granular materials}

\begin{tabular}{|c|c|c|c|c|c|c|c|}
\hline Material & $\begin{array}{l}\text { Density } \rho \\
(g / c c)\end{array}$ & $\begin{array}{l}\text { Diameter } \\
(\mathrm{mm})\end{array}$ & $\begin{array}{l}\text { Young modulus } \\
\left(10^{9} \mathrm{~N} / \mathrm{m}^{2}\right)\end{array}$ & \multicolumn{2}{|c|}{ Coefficient of restitution* } & \multicolumn{2}{|c|}{$\begin{array}{l}\text { Mass of grains } m_{i} \text { used } \\
\text { in the experiments }(g)\end{array}$} \\
\hline Steel beads (S) & $7.8 \pm 0.1$ & $2.0 \pm 0.01$ & $\sim 180$ & \multicolumn{2}{|c|}{$S-S_{b} 0.76 \pm 0.02$} & \multicolumn{2}{|c|}{$\begin{array}{l}1.0,1.5,3.5,7.0,10.5,15.0 \\
18.5,23.5,28.5\end{array}$} \\
\hline Glass beads (G) & $2.6 \pm 0.1$ & $2.0 \pm 0.02$ & $\sim 70$ & \multicolumn{2}{|c|}{$\begin{array}{l}G-S_{b} 0.91 \pm 0.02 \\
G-G_{b} 0.91 \pm 0.01\end{array}$} & \multicolumn{2}{|c|}{$3.5,7.0,10.5$} \\
\hline \multicolumn{8}{|l|}{ Containers } \\
\hline Geometry & & $\begin{array}{l}\text { Dimensions } \\
(\mathrm{cm})\end{array}$ & $\begin{array}{l}\text { Mass } m_{c}(g) \\
\text { (empty) }\end{array}$ & $\begin{array}{l}\text { C. restitution } \varepsilon_{O} \\
\text { (empty) }\end{array}$ & \multicolumn{2}{|c|}{ Filling material } & Drop height $H(\mathrm{~cm})$ \\
\hline $\begin{array}{l}\text { Cylindrical (acrylic tube } \\
\text { with steel ends). }\end{array}$ & $\begin{array}{l}\mathrm{L}=1,2 \\
\mathrm{~L}=35 \\
\mathrm{~L}=3 \\
\mathrm{D} \text { (int/ex }\end{array}$ & $\begin{array}{l}3,4,5,7 \\
t: 1.1 / 1.6\end{array}$ & $\begin{array}{l}10.1 \\
5.810 .8 \\
6.4\end{array}$ & $\begin{array}{l}0.90 \pm 0.02 \\
0.80 \pm 0.02 \\
0.70 \pm 0.03\end{array}$ & \multicolumn{2}{|l|}{$\begin{array}{l}S \\
S, C, G, s \\
S\end{array}$} & $\begin{array}{l}10 \\
20 \\
20\end{array}$ \\
\hline
\end{tabular}

collisions. As a result, the remaining energy of the particles is not enough to reach the container ceiling in the following rebounds, and the particles only hit the container bottom. In oscillating box-type GD, a significant dependence of the damping efficiency on the container length has been found ${ }^{24,25}$. In such experiments, the amplitude of vibration was large enough to produce several interactions of the particles with the ends of the box. However, when the amplitude was reduced, the particles did not have enough energy to reach the box ceiling and they only hit the box bottom, and, as in our results, the box size was also irrelevant ${ }^{24}$. The effect of $m_{i}$ on the total bouncing time (Fig. 2d) is discussed later in the text.

First bounce. The dependence of the coefficient of restitution on the different parameters involved in the experiments is complex. However, this is not the case for the first impact. Because of this reason, let us focus our study in the first rebound, which represents the transition between solid-like to gas-like state for the granular material contained in the ball. The energy of the impact is partitioned between the container and the numerous beads provoking the fast change of state from a granular packing to a granular gas (similar to sublimation ${ }^{21}$ ). The discussion about the clustering process ${ }^{25,35}$ is out of the scope of the present work. However, this represents an interesting system for further investigations in this field.

Figure 3 a shows the values of $\varepsilon$ for the first impact as a function of $m_{i} / m_{c}(\bigcirc)$ obtained from the data reported in Fig. $1 \mathrm{~h}$. Note that three different regimes are observed: (I) for $0<m_{i} / m_{c}<1.5, \varepsilon$ decreases linearly from a quasi-elastic collision $\left(\varepsilon_{0}=0.9\right)$ to almost zero. (II) For $1.5<m_{i} / m_{c}<2.3, \varepsilon \approx 0$ indicating a completely inelastic collision, and (III) for $m_{i} / m_{c}>2.3, \varepsilon$ starts to augment again and a small increment in mass produces a big increment in the coefficient of restitution. The linear decrease indicates that a percentage of the initial momentum of the container is transferred during the impact to the grains, and this transfer augments with the number of particles. The transition at $m_{i} / m_{c} \approx 1.5$ indicates the minimum mass of grains

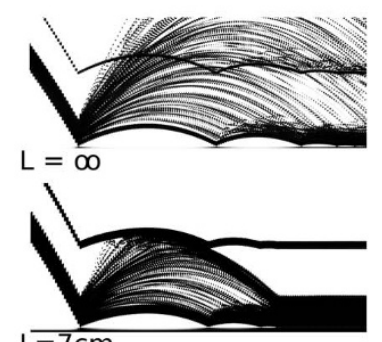

$\mathrm{L}=7 \mathrm{~cm}$

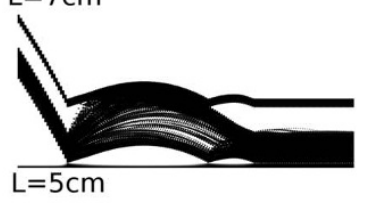

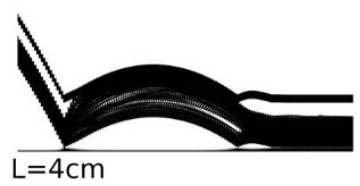
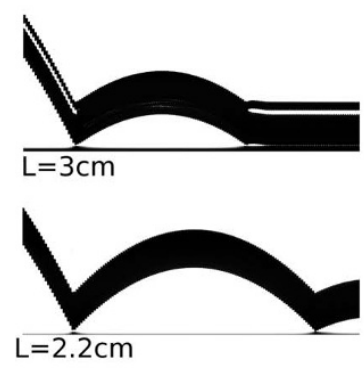

a)

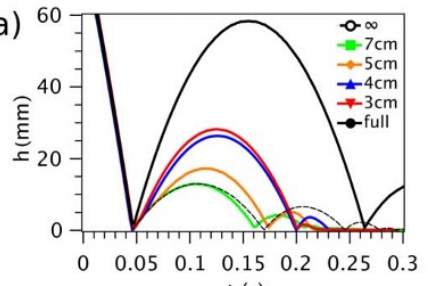

b)

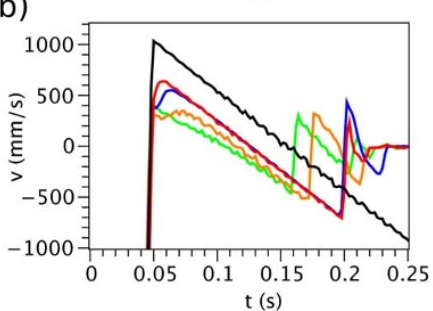

c)

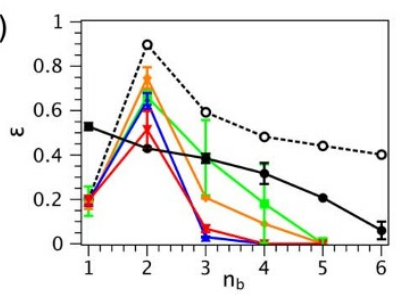

d)

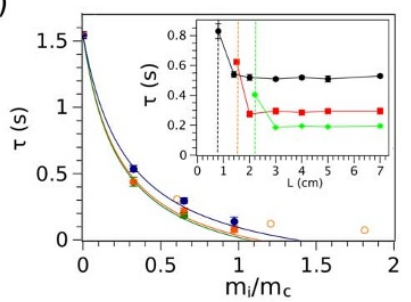

Figure $2 \mid$ Snapshots of the bouncing dynamics of cylindrical containers of different lengths L. (a) $h v s t$, (b) $v v s t$ and (c) $\varepsilon v s n_{b}$. Colours are in correspondence with (a). (d) $\tau v s m_{i} / m_{c}$ for steel $(\bullet)$, ceramic $(\bigcirc)$ and glass $(\bigcirc)$. Only for comparison, the time of the particles motion is shown $(\mathrm{O})$. Inset: for a given mass of grains, $\tau$ is independent on the tube length and only increases when the available space inside the container is completely full of grains. The dotted lines correspond to the space occupied by the grains for $m_{i}=3.5 \mathrm{~g}(\bullet), 7 \mathrm{~g}(\boldsymbol{\square})$, and $10.5 \mathrm{~g}(\diamond)$. 

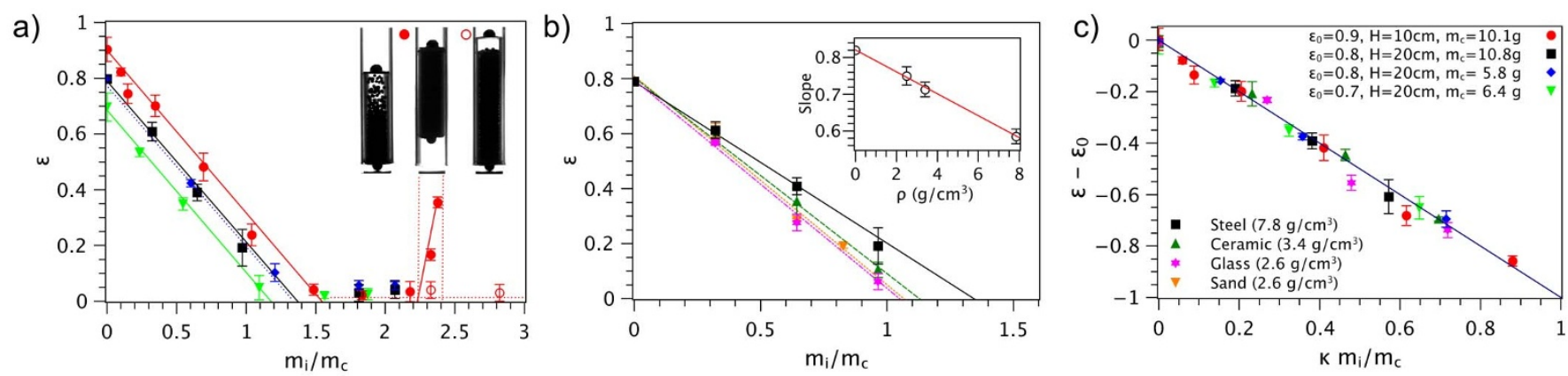

Figure $3 \mid$ (a) Restitution coefficient $\varepsilon$ vs $m_{i} / m_{c}$ for the first bounce of a cylinder with steel particles and $\varepsilon_{0}=0.9(\boldsymbol{\bullet}, \boldsymbol{\bullet}), \varepsilon_{0}=0.8(\boldsymbol{\square}, \diamond)$, and $\varepsilon_{0}=$ $0.7(\nabla) .(b) \varepsilon$ vs $m_{i} / m_{c}$ for cylinders with different granular materials: $\operatorname{steel}(\boldsymbol{\square})$, ceramic $(\boldsymbol{\Delta})$ and glass $\left({ }^{*}\right)$. Here, $\varepsilon_{0}=0.8$ for the three cases. $(\mathrm{c}) \varepsilon-\varepsilon_{0}$ vs $m_{i} / m_{c}$ for all the above conditions. The legend shows all the details about $m_{i}, m_{\mathcal{c}}, \varepsilon_{0}, H$ and granular materials used in (a-c). The solid black line corresponds to the best fit of equation (1).

$m_{\text {crit }}$ necessary to remove all the initial momentum of the container. Over this critical value, the collision is completely inelastic. The sudden increase of $\varepsilon$ observed at $m_{i} / m_{c}=2.3$ occurs because the available internal space inside the container $\left(V=4.75 \mathrm{~cm}^{3}\right)$ becomes full of particles for this mass value. Then, the particles do not have enough room to move and the damping is less efficient. Considering the random close packing $\varphi=0.64$, the maximum mass is $m_{i}=\rho \varphi V$ $\approx 23.5 \mathrm{~g}$, which corresponds to $m_{i} / m_{c}=2.3$. When the tube was tapped to increase the packing, a small free space was created and some particles were added to fill the empty space. A new measurement of $\varepsilon$ increases from 0.15 to 0.4 by only adding 10 particles. This indicates that, for the highest packing of grains, the bouncing dynamics approaches to the behaviour of a solid object. To show that the coefficient of restitution augments because of the lack of free space and not due to the mass, a longer container (with same $m_{c}$ ) with a larger inner mass of particles $\left(m_{i}=28.5\right.$ g, i.e. $\left.m_{i} / m_{c}=2.8\right)$ was used, obtaining again $\varepsilon=0$, see (o) in Fig. $3 a$.

The experiments were repeated using containers with different coefficients of restitution $\left(\varepsilon_{0}=0.8 \boldsymbol{\square}\right.$, and $\left.\varepsilon_{0}=0.7 \nabla\right)$ released from $\mathrm{H}=20 \mathrm{~cm}$, see Fig. 3a. In all cases, $\varepsilon$ decreases linearly with $m_{i} / m_{c}$ from $\varepsilon_{0}$ to practically zero at a given critical mass. This behaviour suggests a linear fit of the form: $\varepsilon=\varepsilon_{0}-k m_{i} / m_{c}$. In so doing, we found that the fitted slope $k$ is the same for the four sets of experiments, which are only shifted by the intercept $\varepsilon_{0}$. On the other hand, the slope varies slightly when the granular material is changed $(k=$ $0.58,0.71$ and $0.75 \pm 0.02$ for steel, ceramic and glass beads, respectively), see fig. $3 \mathrm{~b}$. Then, $k$ must be related to the material properties. The inset in Fig. $3 \mathrm{~b}$ shows that $k$ decreases linearly with the density of (i)

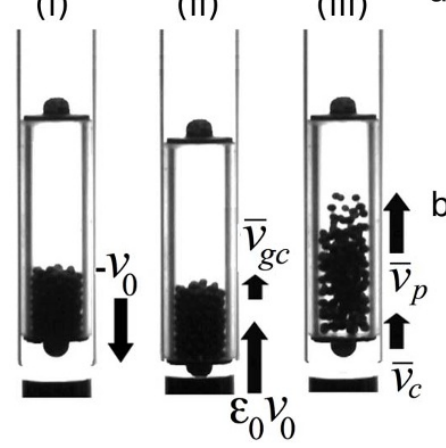

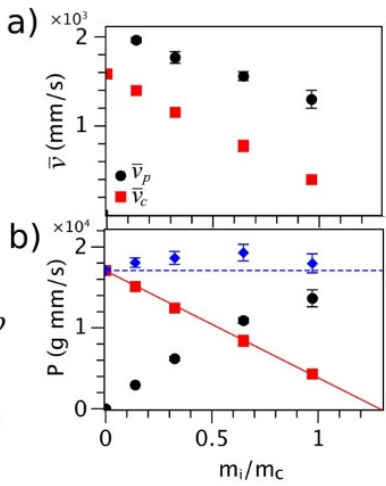

Figure $4 \mid$ (i-iii) Stages considered in the momentum transfer model. (a) Velocity $v$ and (b) momentum $P$ of the grains $(\mathbf{O})$ and container $(\boldsymbol{\square})$ for different masses of steel particles. The total momentum of the system is also $\operatorname{shown}(\diamond)$. For these data, the container $\left(\varepsilon_{0}=0.8, m_{c}=10.8 \mathrm{~g}\right)$ was dropped from $H=20 \mathrm{~cm}$. the grains $\rho$. This fact can be explained considering the energy dissipation capacity ${ }^{24}$. For a given mass $m_{i}$, the number of particles $N$ is inversely proportional to $\rho$ (e.g., if $m_{i}=10.5 \mathrm{~g}, N_{s} \approx 320, N_{c} \approx 740$, and $N_{g} \approx 960$ ). A larger number of particles implies more collisions, and therefore higher energy dissipation. According to this fit, all the above data were summarized in the plot $\varepsilon-\varepsilon_{0}$ vs $k m_{i} / m_{c}$ (Fig. 3c). Note that the collapse is remarkable.

\section{Discussion}

Let us now analyse the momentum conservation of the containerparticles system during the first bounce. It is difficult to manage momentum exchange systems that involve simultaneous con$\operatorname{tacts}^{36-40}$. Indeed, the forces acting at the contact points between the grains are strongly interdependent; therefore, the calculation of the momentum distribution is complex. In this problem, we propose to decompose the process in three steps (Fig. 4): (i) First, we consider the container and grains as two separated systems impacting the base
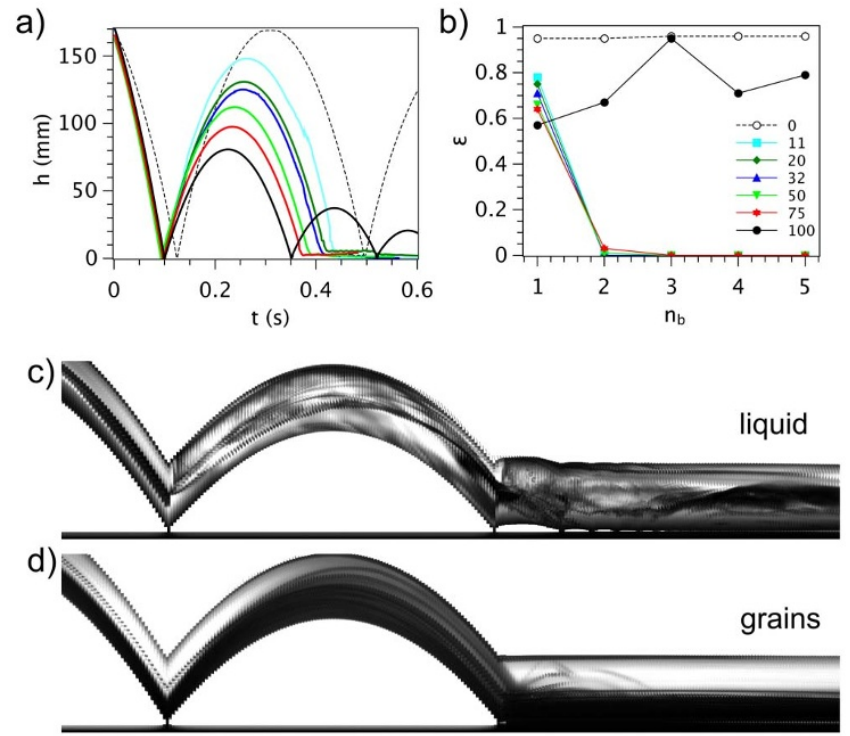

Figure 5 $\mid$ Bouncing dynamics of a sphere partially filled with different volumes of grains $V_{f}$ : (a) $h$ vs $t$ and (b) $\varepsilon v s n_{b}$ for $V_{f} / V_{\text {sphere }}=0(\bigcirc)$, $0.11(\square), 0.20(\diamond), 0.32(\boldsymbol{\Delta}), 0.50(\nabla), 0.75\left(^{*}\right)$ and 1( $)$. The damping of the first rebound increases with the volume of grains due to the deformation of the ball, but a zero rebound never occurs. However, the second collision is completely inelastic in all cases. These results are in clear contrast with the liquid-filled sphere behaviour, where the maximum damping is obtained with a liquid volume of $30 \%$. (c-d) Montages showing the rebound of a sphere partially filled with $15 \mathrm{~g}$ of (c) water and (d) grains. 


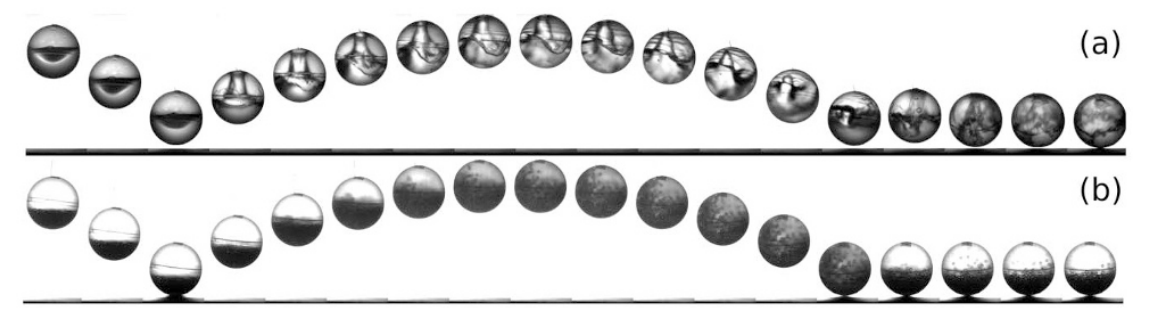

Figure 6 Snapshots of the bouncing dynamics of a sphere partially filled with $\mathbf{1 5} \mathrm{g}$ of (a) water and (b) grains. These images allow us to observe the motion of the internal material. The liquid reacts violently to the impact and forms a jet inside the sphere. Note that the liquid is always in contact with the container, in contrast with the "granular gas" decoupled from the inner surface of the sphere. The abrupt collapse of the grains dissipates all the energy of the system during the second impact.

with a velocity $v_{0}$. (ii) In so doing, the vertical velocity of the container after the collision is $\varepsilon_{0} v_{0}$ and the average velocity of the grain cluster is $\left\langle v_{g c}\right\rangle$. Here, it is assumed that $\left\langle v_{g c}\right\rangle \ll \varepsilon_{0} v_{0}$ due to the fast energy dissipation in the granular material. (iii) Then, the empty container transfers part of its momentum to the grains via a wave front of collisions travelling through the cluster. Finally, the container emerges with a velocity $v_{c}$ and the particles with an average velocity $\left\langle v_{p}\right\rangle$. In Fig. $4 \mathrm{a},\left\langle v_{p}\right\rangle$ and $v_{c}$ are shown as function of $m_{i} / m_{c}$. Multiplying these velocities by $m_{i}$ and $m_{c}$, respectively, one obtains the momenta $\mathrm{P}$ after the collision, see Fig. $4 \mathrm{~b}$. Note that the momentum of the container decreases whereas the average momentum of the grains increases, as a result of the momentum exchange. Applying the momentum conservation we have: $m_{c} \varepsilon_{0} v_{0}$ $+m_{i}\left\langle v_{g c}\right\rangle=m_{c} v_{c}+m_{i}\left\langle v_{p}\right\rangle$. Since $\varepsilon=v_{c} / v_{0}$, this expression reduces to:

$$
\varepsilon=\varepsilon_{0}-\left(\Delta v_{p} / v_{0}\right) m_{i} / m_{c}
$$

where $\Delta v_{p}=\left\langle v_{p}\right\rangle-\left\langle v_{g c}\right\rangle$. Let us remind that equation (1) expresses the momentum transfer: $m_{c} \Delta v_{c}=m_{i} \Delta v_{p}$. The above model describes accurately the experimental data shown in Fig. 3. It is noteworthy that $\Delta v_{p} / v_{0}=k$ remains constant for a given material. This means that $\varepsilon$ is independent of the impact velocity. Moreover, from equation (1), one finds that the critical mass to obtain a completely inelastic collision is given by:

$$
m_{\text {crit }}=\varepsilon_{0} m_{c} / k
$$

Considering that $k \sim 0.58$ to 0.75 for the wide range of granular materials densities analysed $\left(\sim 2-8 \mathrm{~g} / \mathrm{cm}^{3}\right)$, one can approximate $m_{\text {crit }} \sim 1.5 \varepsilon_{0} m_{c}$. In summary, the knowledge of the mass of the empty container and its coefficient of restitution are, in a first approximation, sufficient to determine the amount of granular material necessary to obtain a zero rebound, independently of the properties of the granular material. This outstanding conclusion is similar to the universal response of optimal granular dampers found recently using numerical simulations ${ }^{27}$ : whenever a large number of particles is used, the total energy dissipated (collisional and frictional) is largely independent of the friction and restitution characteristics of the particles.

An expression for the total bouncing time can be derived using $m_{\text {crit }}$. In Fig. $2 \mathrm{~d}$, it was shown that $T$ decreases notably as $m_{i}$ increases. Given that $T\left(m_{i}=0\right)=T_{0}$ and $T\left(m_{\text {crit }}\right)=0$, a plausible dependence for $T\left(m_{i}\right)$ can be written as follows: $T\left(m_{i}\right)=T_{0}\left(1-m_{i} / m_{\text {crit }}\right) /(1+$ $\left.\beta m_{i} / m_{\text {crit }}\right)$, where $\beta$ is a free parameter. This function was plotted in Fig. $2 \mathrm{~b}$ (solid lines) using equation (2) with $\varepsilon_{0}=0.8$ and the values of $k$ found previously for steel, ceramic and glass particles. Again, the good agreement with the experimental measurements supports the momentum transfer model.

Finally, we compared briefly the previous results with the ones obtained using a sphere partially filled with different masses of grains. Unlike the cylindrical geometry, we found that the first bounce of the sphere was largely unaffected by the internal material (as in the case of a liquid-filled sphere ${ }^{10}$ ), however, the second impact is totally inelastic. This completely inelastic bounce was observed when $m_{i} \gg m_{c}$ for different filling volumes $V_{f}$ (see Fig. 5a). Only the sphere completely full of grains and the empty sphere bounce several times before a complete stop. For partially filled spheres the coefficient of restitution is always zero at the second impact, see Fig. 5b. It is important to clarify that the damping of the first rebound increases with $V_{f}$ due to the larger deformation of the ball, as was corroborated using spheres with equivalent mass of liquid. Nevertheless, following the second impact, the liquid-filled sphere displays a series of small consecutive bounces, whereas the grainfilled sphere stops dramatically, see Figs. 5c-d. Snapshots in Fig. 6 reveal that the liquid reacts more quickly to the sphere impact than the grains. However, whereas the liquid is always in contact with the sphere surface, the grains are decoupled from the sphere and form a granular gas that collapses at the second impact. This abrupt collapse dissipates all the energy of the system. In contrast, the water continues splashing inside the other sphere which remains rebounding. Additional research in this regard and the role of confinement produced by the geometry will be presented in a future work.

\section{Methods}

Cylindrical containers. Transparent acrylic tubes of different lengths $L$ were closed with rounded steel caps (a sphere of $6 \mathrm{~mm}$ welded to a washer of $14 \mathrm{~mm}$ diameter). Each tube was filled with a given mass $m_{i}$ of small spherical particles. Then, the container was released from a given height $H$ over a massive horizontal steel base, see details in Table 1. A glass tube was used to maintain the projectile aligned vertically, as is shown in Fig. 1a. The projectile impacts and bounces over the solid surface until reach the repose. The experiments were filmed with a high speed camera at $1000 \mathrm{fps}$ and the videos were analysed using ImageJ to obtain the dynamics. The experiments were repeated five times for the following parameters: $m_{i}, L, \mathrm{~m}_{\mathrm{c}}, \varepsilon_{0}, \rho_{\text {grains }}$ and $v_{0}$ (see details in Table 1). On the other hand, solid steel bars of equivalent masses $m_{i}$ were glued into the container. By impacting these projectiles, we found that $\varepsilon_{0}$ does not change significantly (less than 5\%) in the range of masses used in the experiments. Then, the results reported here are due to the grains-container interaction and not to an effect of the total mass of the projectile. Moreover, using free-fall experiments, the air influence was checked to be negligible on the container motion and on the motion of one simple particle.

Spherical containers. Transparent celluloid ping-pong balls were filled with different masses of steel beads or water. The spheres were dropped from $H=20 \mathrm{~cm}$ and filmed at $1000 \mathrm{fps}$. The analysis was similar to the used with the cylindrical geometry.

Montages. The snapshots in Figs. 2 and 5 were formed by combining a 10 pixels width line from the centre of the container from each image of the high speed videos.

1. Barnes, G. Study of collisions Part I. A survey of the periodical literature. Am. J. Phys. 26, 5-8 (1958).

2. Bayman, B. F. Model of the behaviour of solid objects during collision. Am. J. Phys. 44, 671-676 (1976)

3. Falcon, E. et al. Behavior of one inelastic ball bouncing repeatedly o the ground. Eur. Phys J. B 3, 45-57 (1998).

4. Cross, R. The bounce of a ball. Am. J. Phys. 67(3), 222-227 (1999).

5. Kanso, E. \& Papadopoulos, P. Dynamics of pseudo-rigid ball impact on rigid foundation. Int. J. Non-Linear Mech. 39, 299-309 (2004).

6. Nagurka, M. \& Huang, S. A. Mass-Spring-Damper Model of a Bouncing Ball. Int. J. Eng. 22(2), 393-401 (2006).

7. Bernu, B. \& Mazighi, R. One-dimensional bounce of inelastically colliding marbles on a wall. J. Phys. A 23, 5745 (1990). 
8. Ramírez, R. et al. Coefficient of restitution of colliding viscoelastic spheres. Phys. Rev. E 60, 4465 (1999).

9. Carre, M. J. et al. Impact of a non-homogeneous sphere on a rigid surface. J. Mech. Eng. Sci. 218(3), 273-281 (2004).

10. Gondret, P. et al. Bouncing motion of spherical particles in fluids. Phys. Fluids $\mathbf{1 4}$ 643-652 (2002).

11. Mehta, A. \& Luck, J. M. Novel Temporal Behavior of a Nonlinear Dynamical System: The Completely Inelastic Bouncing Ball. Phys. Rev. Lett. 65, 393-396 (1990)

12. Dorbolo, S. et al. Dynamics of a bouncing dimer. Phys. Rev. Lett. 95, 044101 (2005).

13. Dorbolo, S. et al. Bouncing trimer: a random self-propelled particle, chaos and periodical motions. New J. Phys. 11, 033016 (2009).

14. Luding, S. et al. Studies of columns of beads under external vibrations. Phys. Rev. E 49, 2 (1994).

15. Umbanhowar, P. B. et al. Localized excitations in a vertically vibrated granular layer. Nature 382, 793-796 (1996).

16. Kudrolli, A. et al. Cluster Formation due to Collisions in Granular Material. Phys. Rev. Lett. 78, 7 (1997).

17. Pacheco-Vázquez, F., Caballero-Robledo, G. A. \& Ruiz-Suárez, J. C. Superheated granular matter. Phys, Rev. Lett. 102, 170601 (2009).

18. Cross, R. Dynamic properties of tennis balls. Sports Engineering 2, 23-33 (1999).

19. Mars Exploration Rovers. Step by Step Guide to Entry, Descent and Landing. Jet Propulsion Laboratory, California Institute of Technology (2013). http:// marsrover.nasa.gov/mission/tl_entryl.html.

20. Killian, T. W. et al. Rebound and jet formation of a fluid-filled sphere. Phys. Fluids 24, 122106 (2012)

21. Salueña, C., Pöschel, T. \& Esipov, S. E. Dissipative properties of vibrated granular materials. Phys. Rev. E 59, 4422-4425 (1999).

22. Sato, T. et al. Vibration isolation in a system using granular medium. JSME 38 C(3), 434-440 (1995).

23. Mao, K. et al. Simulation and characterization of particle damping in transient vibrations. J. Vib. Acoust. 126, 202-211 (2004).

24. Bai, X-M. et al. Investigation of particle damping mechanism via particle dynamics simulations. Granular Matter 11, 417-429 (2009).

25. Bannerman, M. N. et al. Movers and shakers: Granular damping in microgravity. Phys. Rev. E 84, 011301 (2011).

26. Sánchez, M. \& Pugnaloni, L. A. Effective mass overshoot in single degree of freedom mechanical systems with a particle damper. J. of Sound and Vibration 330, 5812-5819 (2011)

27. Sánchez, M., Rosenthal, G. \& Pugnaloni, L. A. Universal response of optimal granular damping devices. J. of Sound and Vibration 331, 4389-4394 (2012).

28. Panossian, H. V. Structural damping enhancement via non-obstructive particle damping technique. J. Vib. Acoust. 114(1), 101-105 (1992).

29. Rubin, S. \& Oaks, S. Tuned broadband particulate vibration absorber. US Patent 5855260A (1999).

30. Ashley, S. “A New Racket Shakes Up Tennis”. Mech. Eng. 117, 80-81 (1995).

31. Pöschel, T. et al. Hand-Held Medical Instrument. US Patent 20100331828.
32. Heckel, M. et al. Granular dampers for the reduction of vibrations of an oscillatory saw. Physica A 391, 4442-4447 (2012).

33. Norcross, J. C. Dead-blow hammer head. US Patent 3343576.

34. Cheng, T. et al. Dissipation mechanisms of nonobstructive particle damping using discrete element method. Proc. SPIE 4331, 294-301 (2001).

35. Opsomer, E., Ludewig, F. \& Vandewalle, N. Phase transitions in vibrated granular systems in microgravity. Phys. Rev. E 84, 051306 (2011).

36. Liu, C. et al. Frictionless multiple impacts in multibody systems. Part I. Theoretical framework. Proc. R. Soc. A 464(2100), 3193-3211 (2008).

37. Liu, C. et al. Energy dissipation and dispersion effects in a granular media. Phys. Rev. E 78(031307), 1-13 (2008)

38. Liu, C. et al. Variable structure dynamics in a bouncing dimer. INRIA Research Report 6718, (2008).

39. Liu, C. et al. Frictionless multiple impacts in multibody systems. Part II. Numerical algorithm and simulation results. Proc. R. Soc. A 465(2101), 1-23 (2009).

40. Zhao, Z. et al. Planar dynamics of a rigid body system with frictional impacts. Part II. Qualitative analysis and numerical simulations. Proc. R. Soc. A 465(2107), 2267-2292 (2009).

\section{Acknowledgments}

We thank F. Moreau for his valuable comments. We also thank to N. Vandewalle and GRASP for financial support, help and fruitful discussions. FPV is a beneficiary of a mobility grant from BELSPO/Marie Curie at the Université de Liège. SD thanks to F.R.S.-FNRS and FPV to Conacyt Mexico for financial support.

\section{Author contributions}

F.P.V. carried out the experiments and analysed the data. Both authors discussed the results. F.P.V. wrote the manuscript. Both authors reviewed the manuscript. S.D. supervised the research.

\section{Additional information}

Supplementary information accompanies this paper at http://www.nature.com/ scientificreports

Competing financial interests: The authors declare no competing financial interests.

How to cite this article: Pacheco-Vázquez, F. \& Dorbolo, S. Rebound of a confined granular material: combination of a bouncing ball and a granular damper. Sci. Rep. 3, 2158; DOI:10.1038/srep02158 (2013).

(7) This work is licensed under a Creative Commons Attributionc. visit http://creativecommons.org/licenses/by-nc-sa/3.0 
SUBJECT AREAS:

MECHANICAL ENGINEERING APPLIED PHYSICS AEROSPACE ENGINEERING NONLINEAR PHENOMENA

\section{SCIENTIFIC REPORTS:}

$3: 2158$

DOI: $10.1038 /$ srep02158

(2013)

Published:

9 July 2013

Updated:

15 August 2013
ERRATUM: Rebound of a confined granular material: combination of a bouncing ball and a granular damper

F. Pacheco-Vázquez \& S. Dorbolo

The Supplementary Movie that accompanies this study was omitted from the original version of this Article. 